\section{Removal of Coomassie Blue Precipitates from Polyacrylamide Gels}

\author{
BioTechniques 21:820 (November 1996)
}

During staining of polyacrylamide gels, varying amounts of Coomassie blue may precipitate on the surface of the gels. This background obscures faint bands and is esthetically unappealing. The problem is worse when gels are stained for longer periods of time or when the stain has not been recently filtered.

Rinsing a gel with methanol efficiently and quickly removes this background. Basically, at any time during destaining, gels are treated for $1 \mathrm{~min}$ in $100 \%$ methanol and returned to the destain or water. Gentle shaking is required. If the methanol rinse is done before excess stain is completely removed, it is easier to judge when destaining is complete (because of the lower background). During the treatment, gels may turn opaque, although this does not appear to harm the gel.

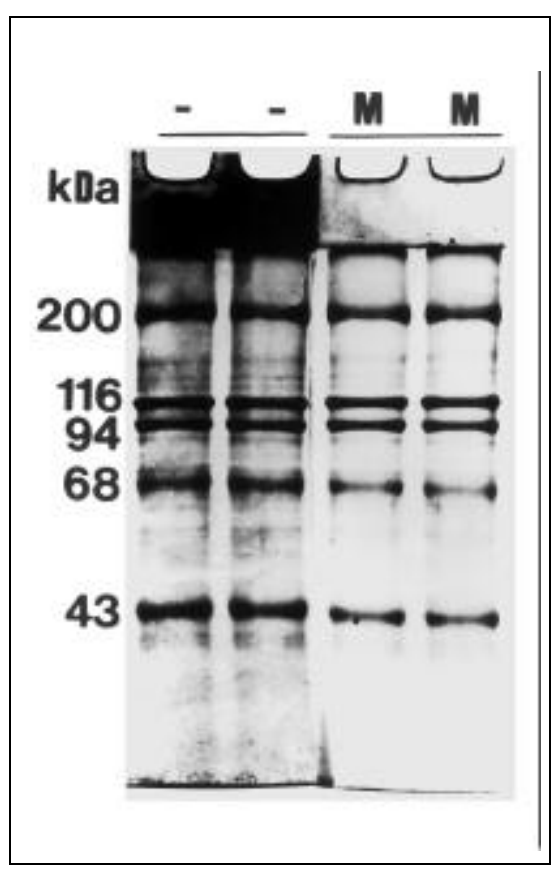

Figure. 1 Coomassie blue-stained polyacrylamide gel. Molecular weight standards (Bio-Rad) were run in each lane. Lanes marked $\mathrm{M}$ were rinsed for 1 min with methanol while the other lanes were kept in destain. Gel was photographed through a yellow filter (Wratten No. 15).
For illustration, a 0.75 -mm-thick sodium dodecyl sulfate $10 \%$ polyacrylamide gel (1) was stained with $0.25 \%$ Coomassie Brilliant Blue $\mathrm{R}^{\circledR}-250$ (BioRad, Hercules, CA. USA) in $25 \%$ isopropanol and $10 \%$ acetic acid for $24 \mathrm{~h}$ and then destained in $7 \%$ acetic acid with four changes of destain over $24 \mathrm{~h}$ (2). The gel was cut in half. One half was kept in destain, while the other was rinsed in methanol for 1 min (Figure 1), as described above.

Ethanol and acetone removed precipitated Coomassie stain at about the same rate as methanol. Isopropanol was slightly less effective. Neither $0.75-\mathrm{mm}$ gels nor $1.5-\mathrm{mm}$ gels (10\% polyacrylamide) appeared to be harmed by 5 min treatments with $100 \%$ methanol, although the stain came off the gel surface in less than one minute. Protracted incubation will remove stain from protein bands. Also, 0.75-mm 5\% and $20 \%$ polyacrylamide gels appeared unharmed by the methanol treatment.

\section{REFERENCES}

1.Laemmli, U.K. 1970. Cleavage of structural proteins during the assembly of the head of the bacteriophage T4. Nature 227:680-685.

2.Promega Corporation. Promega Protocols and Applications Guide. 1991. 2nd ed., p. 255. David E. Titus (Ed.). Madison, WI.

This work was supported by the U.S. Naval Medical Research and Development Command, Bethesda, MD, work unit No. B690.00101.EOX.3418. The opinions and assertions contained here are the private ones of the author and are not to be construed as official or reflecting the views of the Navy Department or the U.S. Government. Address correspondence to Samuel A. Lewis, Research Publications Branch, NAM$R U 3$ United States Naval Medical Research Unit, PSC 452, FPO, AE 09835, USA. Internet:namru3@wrair-emh1.army.mil

Received 5 July 1995; accepted 14 March 1996.

\section{Samuel A. Lewis}

United States Naval Medical

Research Unit 3

Code $101 F$

FPO, AE, USA
Modified Crush-and-Soak Method for Recovering Oligodeoxynucleotides from Polyacrylamide Gel

BioTechniques 21:820-822 (November 1996)

Oligodeoxynucleotides are often purified by polyacrylamide gel electrophoresis (PAGE). The two commonly used methods for recovering oligodeoxynucleotides (ODN) from polyacrylamide gels are the electroelution and the crush-and-soak methods (1). Electroelution is fast but inconvenient, as it requires a special apparatus. The crush-and-soak method is simple but time-consuming. In a standard crushand-soak method, a gel fragment containing the ODN is excised, crushed and soaked overnight in sodium acetate buffer to extract the oligodeoxynucleotide. Here, we describe a modified crush-and-soak method that eliminates the overnight soaking yet gives comparable yields.

For our method, the ODN-containing gel fragment is swollen at $90^{\circ} \mathrm{C}$ for $5 \mathrm{~min}$, frozen at $-70^{\circ} \mathrm{C}$ for $5 \mathrm{~min}$, and ODN-recovered by centrifugation. We tested this approach for the purification of a 22-nucleotide ODN and obtained yields comparable to those using an overnight soak. The ODN used in this comparison had previously been purified by PAGE so that there would be no truncated ODN, unincorporated nucleotides and cleaved protecting groups that might interfere with the spectrophotometric analysis of the ODN stock solution. Eight aliquots each containing $10 \mu \mathrm{L}$ of $33 \mu \mathrm{M}$ ODN and $10 \mu \mathrm{L}$ of $0.01 \%$ bromophenol blue in $95 \%$ formamide were loaded and run into a $20 \%$ denaturing polyacrylamide gel. Following electrophoresis, the ODN was identified by ultraviolet (UV) shadowing with a handheld short-wavelength UV lamp. The ODN-containing bands could be easily seen under UV light with the gel still attaching to the lower glass gel plate (regular window glass) against a black background. The target ODN band was excised with a sharp scalpel and placed in a column filter (Quick-Snap Column ${ }^{\circledR}$; IsoLab, Akron, OH, USA). The gel slices 